\title{
Effect of Allogeneic Intraoperative Blood Transfusion on Survival in Patients Treated With Radical Cystectomy for Nonmetastatic Bladder Cancer: Results From a Single High-Volume Institution
}

\author{
Marco Moschini, ${ }^{1,2}$ Paolo Dell' Oglio, ${ }^{1}$ Paolo Capogrosso, ${ }^{1}$ Vito Cucchiara, ${ }^{1}$ \\ Stefano Luzzago, ${ }^{1}$ Giorgio Gandaglia, ${ }^{1}$ Fabio Zattoni, ${ }^{3}$ Alberto Briganti, ${ }^{1}$ \\ Rocco Damiano, ${ }^{2}$ Francesco Montorsi, ${ }^{1}$ Andrea Salonia, ${ }^{1}$ Renzo Colombo ${ }^{1}$
}

\begin{abstract}
Transfusion has been related to poor survival after surgery in several cancers. Recently, timing of transfusion has been proposed as crucial in the determination of poor survival expectanies after surgery, in fact, intraoperative but not postoperative transfusion were found to be related. We confirmed these findings in patients who underwent radical cystectomy because of bladder cancer; physicians should avoid use of transfusion intraoperatively.

Background: Previous studies have demonstrated that perioperative blood transfusion (BT) is associated with a significantly increased risk of cancer recurrence and mortality after radical cystectomy (RC). Recently, it was shown for the first time that intraoperative transfusion has a detrimental effect on cancer survival. The aim of the current study was to validate this finding in a single European institution. Patients and Methods: The study focused on 1490 consecutive nonmetastatic bladder cancer patients treated with RC at a single tertiary care referral center between January 1990 and August 2013. Kaplan-Meier analyses and Cox regression analyses were used to assess the effect of timing of BT administration (no transfusion vs. intraoperative transfusion vs. postoperative transfusion vs. intraoperative and postoperative transfusion) on cancer-specific mortality (CSM), overall mortality (OM), and disease recurrence. Results: Mean age at the time of RC was 67 years. Overall, $322(21.6 \%)$ patients received intraoperative BT and 97 (6.5\%) received postoperative BT. At a mean follow-up time of 125 months (median, 110 months), the 5- and 10-year CSM rate was 846 (58\%) and 715 (48\%), respectively. In multivariable analyses patients who received intraoperative BT had greater risk of disease recurrence (hazard ratio $[\mathrm{HR}], 1.24 ; P<.04)$, CSM (HR, 1.60; $P<.02$ ), and $\mathrm{OM}(\mathrm{HR}, 1.45 ; P<.03)$. Conversely, this effect disappears with postoperative BT (all $P>$.2). Conclusion: Our study confirms that intraoperative, but not postoperative BT, are related to a detrimental effect on survival after RC. These results should be take into account by physicians to administer BT using the correct timing.
\end{abstract}

Clinical Genitourinary Cancer, Vol. 13, No. 6, 562-7 (c) 2015 Elsevier Inc. All rights reserved.

Keywords: Bladder cancer, Cancer specific mortality, Intraoperative transfusion, Radical cystectomy

\section{Introduction}

Radical cystectomy (RC) with pelvic lymph node (LN) dissection (PLND) is the treatment of choice for muscle-invasive and high-risk

${ }^{1}$ Department of Urology, Urological Research Institute, Vita-Salute University, San Raffaele Scientific Institute, Milan, Italy

${ }^{2}$ Doctorate Research Program, Magna Græcia University of Catanzaro, Catanzaro, Italy

${ }^{3}$ Department of Urology, University of Padua, Padua, Italy nonmuscle invasive bladder cancer (BCa) patients. ${ }^{1}$ However, the 5-year overall life expectancy after surgery is not optimal ranging from $42 \%$ to $58 \%$, according to stage of the disease and node

Submitted: Mar 21, 2015; Revised: Apr 13, 2015; Accepted: Apr 26, 2015; Epub: May 4, 2015

Address for correspondence: Marco Moschini, MD, Department of Urology,

University "Vita-Salute," San Raffaele Hospital, Via Olgettina, 60-20132 Milan, Italy Fax: +02-26435659; e-mail contact: marco.moschini87@gmail.com 
status. $^{2}$ In this context, several biochemical or hematological parameters have been described as possible predictors of survival in BCa patients treated with RC..$^{3-7}$ Specifically, perioperative blood transfusion (PBT) has been recently proposed by several investigators as a possible predictor of poor survival expectance after $\mathrm{RC},{ }^{8-11}$ that underlying hematological factors have a direct role in BCa. This effect was known and investigated in several other malignancies, including breast, larynx, colon-rectal, and prostate cancer. ${ }^{12-17}$ Several mechanisms have been proposed to explain this effect, which include immunosuppressive effects from blood transfusion (BT), ${ }^{18}$ a decreased host immunity caused by anesthetics and opioids, ${ }^{19}$ and a release of increased numbers of circulating tumor cells caused by surgical manipulation. ${ }^{20}$ None of the aforementioned studies evaluated the effect of the timing of BT (intraoperative vs. postoperative) in terms of outcomes after RC. In this context, Abel et $\mathrm{al}^{21}$ in a multiinstitutional cohort of 360 patients, assessed whether individuals who received BT during their surgery had better outcomes compared with patients who received BT during their postoperative hospitalization. They validated their results in a validation cohort of $1770 \mathrm{pa}-$ tients, demonstrating that intraoperative transfusion but not postoperative transfusion was associated with lower survival expectances.

In the current study we aimed to assess the effect of intraoperative BT on survival after RC for BCa in a large contemporary European cohort of patients with nonmetastatic BCa treated at a tertiary referral center, to test the hypothesis that the intraoperative BT in our center increased the risk of disease recurrence, cancer-specific mortality (CSM), and overall mortality (OM).

\section{Patients and Methods Study Population}

After institutional review board approval was obtained, we evaluated 1490 consecutive patients with nonmetastatic BCa treated with RC and bilateral PLND at a single tertiary care referral center between January 1990 and August 2013.

\section{Covariates}

Preoperative patient characteristics were evaluated at admission for RC and included: age at time of surgery, sex, body mass index (BMI), Charlson Comorbidity Index ([CCI] which was set to 0 vs. 1 vs. $\geq 2$ ), preoperative hemoglobin $(\mathrm{Hb})$ level, and anemic status. Tumor characteristics included pathological T stage (pT0-T2 vs. pT3 vs. pT4; classified according to the 2009 tumor, node, metastases classification ${ }^{22}$ ), carcinoma in situ, and surgical margin status. Perioperative BT was defined as transfusion of allogeneic red blood cells during RC or in the postoperative hospitalization. Administration of BT was based on the discretion of the treating physicians and stratified according intraoperative or postoperative administration. Anemic status was defined as a preoperative $\mathrm{Hb}$ value recorded as $<13 \mathrm{~g} / \mathrm{dL}$ for men and $<12 \mathrm{~g} / \mathrm{dL}$ for women, according to the World Health Organization (WHO) definition. $^{23}$

Dedicated genitourinary pathologists examined all surgical specimens. All removed LNs were examined for the presence of nodal metastases. LN invasion was invariably defined as $\geq 1$ metastatic LNs. Positive soft tissue surgical margin (PSTSM) was defined as the presence of tumor at inked areas of soft tissue on the RC specimen.

Clinical and radiological follow-up consisted of a baseline visit at 3 to 4 months after surgery. Subsequently, the minimum follow-up consisted of at least 2 annual visits. Examinations included radiological imaging with computed tomography in all patients. In addition to physical examination with laboratory testing, intravenous pyelography, cystoscopy, urine cytology, urethral washings, and bone scan were carried out if indicated.

\section{Statistical Analyses}

Descriptive statistics of categorical variables focused on frequencies and proportions. Means, medians, and interquartile ranges were reported for continuously coded variables. The Mann-Whitney test and $\chi^{2}$ test were used to compare the statistical significance of differences in medians and proportions, respectively.

Our analyses consisted of different steps. First, Kaplan-Meier analyses were used to evaluate the effect of PBT on the recurrence, $\mathrm{OM}$, and CSM rates in the overall population and after stratification according to the timing of PBT. Second, multivariable Cox regression analyses were used to test the relationship between receipt of transfusion and the risk of recurrence, CSM, and OM after adjusting for age, sex, CCI, PSTSM, pathological stage, number of positive nodes, number of removed nodes, adjuvant chemotherapy, and anemia status.

All statistical analyses were performed using SPSS version 22.0 (IBM Corp, Armonk, NY), with a 2-sided significance level set at $P<.05$.

\section{Results \\ Baseline Characteristics}

Clinical and pathological characteristics of patients included in the study are shown in Table 1. Overall, 580 patients (38.9\%) received BT. Of these, 322 individuals (21.6\%) received intraoperative BT, 161 individuals (10.8\%) received intraoperative and postoperative BT, 97 individuals $(6.5 \%)$ received postoperative BT. As expected, patients who received intraoperative and postoperative BT had lower preoperative $\mathrm{Hb}$ values, were more anemic, and lost more blood during surgery (all $P<.001$ ). Moreover, patients who received BT had the higher rate of LNs removed and of positive surgical margin (all $P<.02$ ). Finally, no statistically significant difference was detected in age, BMI, CCI, pathological T stage, Grade (WHO 1973), carcinoma in situ, number of positive nodes, or neoadjuvant or adjuvant chemotherapy, according to the timing of BT administration (all $P>.1$ ).

\section{Survival Estimates}

Mean (median) follow-up was 125.13 (110) months after surgery. Overall, the 5- and 10-year CSM survival-free rates were 58\% and $48 \%$ (Figure 1A). After stratification according to the timing of BT administration (no transfusion vs. postoperative transfusion vs. intraoperative transfusion vs. intraoperative and postoperative transfusion), the 5- and 10-year CSM rates were $64 \%$ and $52 \%$ versus $60 \%$ and $60 \%$ versus $49 \%$ and $42 \%$ versus $50 \%$ and $40 \%$, respectively $(P<.001$; Figure 1B). Moreover, patients who received 
Table 1 Descriptive Statistics of 1490 Patients Treated With RC for BCa Between 1990 and 2013 Stratified According to Administration of PBT

\begin{tabular}{|c|c|c|c|c|c|c|}
\hline Variables & $\begin{array}{c}\text { Overall } \\
(n=1490 ; 100 \%)\end{array}$ & $\begin{array}{l}\text { Intraoperative BT } \\
(\mathrm{n}=322 ; 21.6 \%)\end{array}$ & $\begin{array}{l}\text { Intraoperative and } \\
\text { Postoperative BT } \\
(\mathrm{n}=161 ; 10.8 \%)\end{array}$ & $\begin{array}{l}\text { Postoperative BT } \\
\text { (n = 97; 6.5\%) }\end{array}$ & $\begin{array}{l}\text { No Transfusion } \\
(\mathrm{n}=910 ; 61.1 \%)\end{array}$ & $P$ \\
\hline Age & & & & & & .8 \\
\hline Mean & 67.1 & 67.0 & 68.0 & 66.5 & 67.0 & \\
\hline Median (IQR) & $68(61-74)$ & $68(61-75)$ & $68(63-74)$ & $68(61-74)$ & $68(61-74)$ & \\
\hline Sex & & & & & & .5 \\
\hline Male & $1245(83.6 \%)$ & $260(80.7 \%)$ & $130(80.7 \%)$ & 76 (78.4\%) & 779 (85.6\%) & \\
\hline Female & 245 (16.4\%) & $62(19.3 \%)$ & $31(19.3 \%)$ & $21(21.6 \%)$ & $131(14.4 \%)$ & \\
\hline BMI & & & & & & .8 \\
\hline Mean & 25.6 & 25.7 & 25.8 & 25.5 & 25.4 & \\
\hline Median (IQR) & $25.2(23.4-27.8)$ & $25.3(23.2-27.5)$ & $25.3(23.5-28.0)$ & $24.9(23.9-28.0)$ & $25.1(22.7-28.4)$ & \\
\hline CCI & & & & & & .2 \\
\hline 0 & $525(35.2 \%)$ & $99(30.7 \%)$ & $52(32.3 \%)$ & $41(42.3 \%)$ & $333(36.6 \%)$ & \\
\hline 1 & $549(36.8 \%)$ & $121(37.6 \%)$ & $76(47.6 \%)$ & $36(37.1 \%)$ & $316(34.7 \%)$ & \\
\hline$\geq 2$ & $416(28.0 \%)$ & $102(31.7 \%)$ & $33(20.5 \%)$ & $20(20.6 \%)$ & $261(28.7 \%)$ & \\
\hline \multicolumn{7}{|c|}{ Preoperative $\mathrm{Hb}, \mathrm{mg} / \mathrm{dL}$} \\
\hline Mean & 12.5 & 12.3 & 11.7 & 12.7 & 12.9 & $<.001$ \\
\hline Median (IQR) & $12.6(11.1-13.9)$ & $12.4(11.0-13.8)$ & $11.7(10.4-12.9)$ & $13.0(11.4-14.0)$ & $13.2(11.7-14.3)$ & \\
\hline Anemic Status & 768 (51.5\%) & $208(64.6 \%)$ & $108(67.1 \%)$ & $65(67.0 \%)$ & 387 (42.5\%) & $<.001$ \\
\hline Positive Nodes & & & & & & .2 \\
\hline Mean & 2.4 & 2.6 & 2.8 & 3.3 & 2.2 & \\
\hline Median (IQR) & $0(0-2)$ & $0(0-2)$ & $0(0-2)$ & $0(0-3.5)$ & $0(0-1)$ & \\
\hline Nodes Removed & & & & & & .003 \\
\hline Mean & 18.3 & 18.5 & 18.0 & 22.1 & 17.9 & \\
\hline Median (IQR) & $17(11-24)$ & $16(10-24)$ & $16(10-25)$ & $22(16.0-28.0)$ & $16(11-23)$ & \\
\hline Pathological Stage & & & & & & .3 \\
\hline рT0-pT2 & $650(43.6 \%)$ & $136(42.2 \%)$ & $65(40.4 \%)$ & $42(43.3 \%)$ & $407(44.7 \%)$ & \\
\hline pT3 & 547 (36.7\%) & $121(37.6 \%)$ & $61(37.9 \%)$ & $36(37.1 \%)$ & $329(36.2 \%)$ & \\
\hline pT4 & 293 (19.7\%) & $65(20.2 \%)$ & $35(21.7 \%)$ & $19(19.6 \%)$ & $174(19.1 \%)$ & \\
\hline Grade & & & & & & .3 \\
\hline $1-2$ & 452 (30.3\%) & 93 (28.9\%) & 53 (32.9\%) & $31(32.0 \%)$ & 275 (30.2\%) & \\
\hline $3-4$ & $1038(69.7 \%)$ & 229 (71.7\%) & $108(67.1 \%)$ & $66(68.0 \%)$ & $635(69.8 \%)$ & \\
\hline Blood Loss, cc & & & & & & $<.001$ \\
\hline Mean & 1494 & 1561 & 1779 & 985 & 1031 & \\
\hline Median (IQR) & $1200(800-1800)$ & $1400(962-1900)$ & $1500(1000-2100)$ & $800(500-1400)$ & $800(600-1100)$ & \\
\hline CIS & 307 (20.6\%) & $65(20.2 \%)$ & $33(20.5 \%)$ & $19(19.6 \%)$ & $190(20.9 \%)$ & .9 \\
\hline Surgical Margin & $114(7.7 \%)$ & 31 (9.6\%) & $16(9.9 \%)$ & $9(9.3 \%)$ & $58(6.4 \%)$ & .02 \\
\hline Neoadjuvant CT & $43(2.9 \%)$ & $11(3.4 \%)$ & $6(3.7 \%)$ & $3(3.1 \%)$ & $23(2.5 \%)$ & .1 \\
\hline Adjuvant CT & 384 (25.8\%) & 92 (28.6\%) & $46(28.6 \%)$ & 28 (28.9\%) & $218(24.0 \%)$ & .3 \\
\hline
\end{tabular}

Abbreviations: $\mathrm{BCa}=$ bladder cancer; $\mathrm{BMI}=$ body mass index; $\mathrm{BT}=$ blood transfusion; $\mathrm{CCl}=$ Charlson Comorbidity Index; $\mathrm{ClS}=\mathrm{carcinoma}$ in situ; $\mathrm{CT}=$ chemotherapy; $\mathrm{Hb}=$ hemoglobin; $\mathrm{IQR}=$ interquartile range; $\mathrm{PBT}=$ perioperative blood transfusion; $\mathrm{RC}=$ radical prostatectomy.

intraoperative or intra- and postoperative transfusion had a significantly lower rate of 10-year OM-free rates, compared with patients who received postoperative BT or patients who did not receive BT $(P<.001$; Figure $1 C)$. Similar results were found in an evaluation of 10-year recurrence-free rates $(P=.02$; Figure $1 D)$. No differences were reported in disease recurrence, OM, and CSM rates between intraoperative and intraoperative with postoperative BT (all $P>.3$ ).

\section{Multivariable Cox Regression Analyses in the Overall Population and Stratified According to Anemia Status}

Table 2 shows multivariable Cox regression analyses for assessment of the relationship between receipt of transfusion and the risk of recurrence, CSM, and OM. Because of the absence of a significant difference between patients who received intraoperative BT and patients who received intra- and postoperative BT, we combined these 2 groups in multivariable Cox regression analyses. 

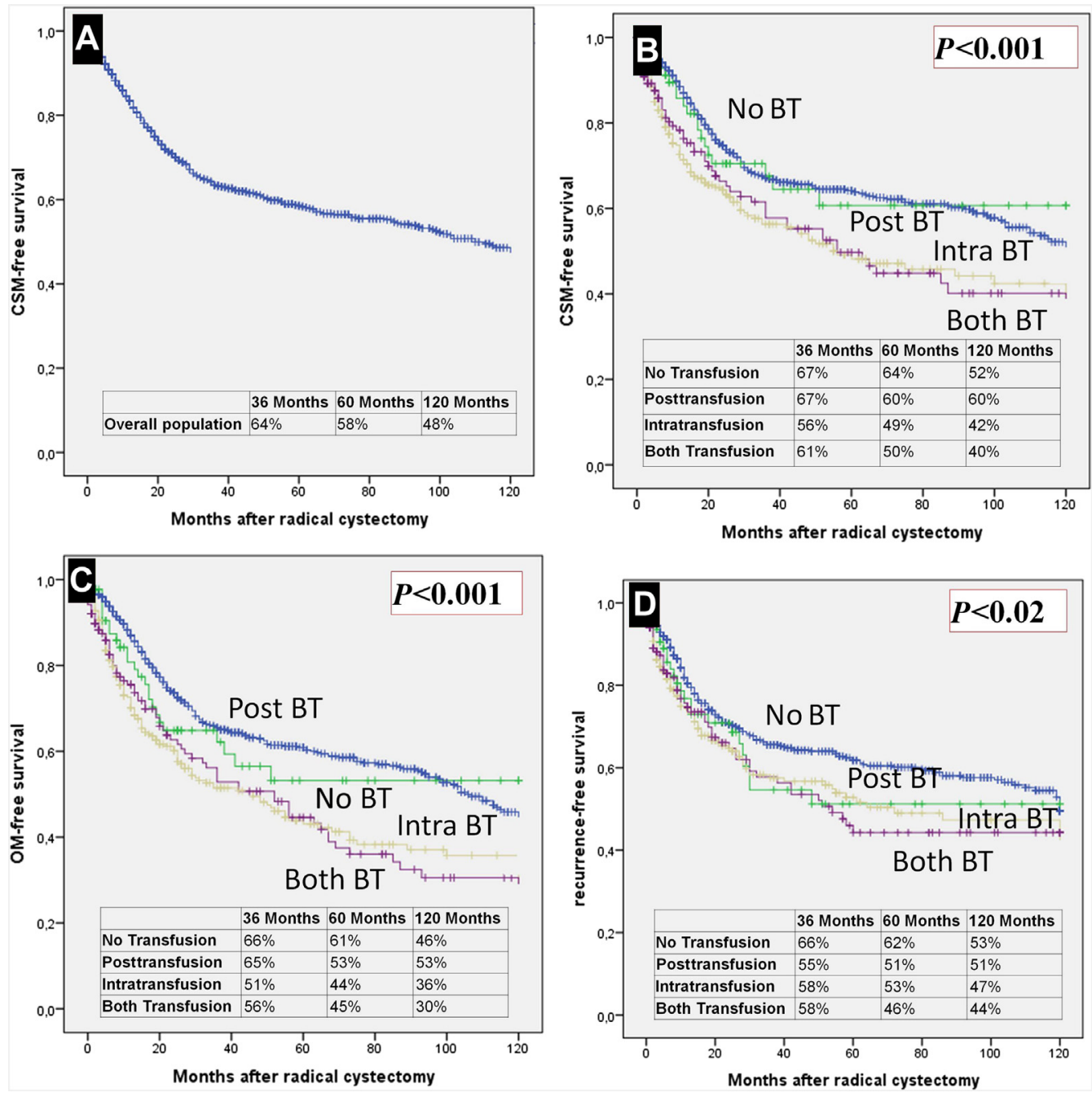

Receipt of postoperative BT was not associated with increased risk of CSM, OM, or disease recurrence (all $P>$.2). However, receipt of intraoperative BT was significantly associated with CSM (hazard ratio $[\mathrm{HR}], 1.60 ; 95 \%$ confidence interval $[\mathrm{CI}], 1.20-2.26 ; P=.02)$, OM (HR, 1.45; 95\% CI, 1.02-2.08; $P=.03$ ), and disease recurrence (HR, 1.24; 95\% CI, 1.03-1.65; $P=.04$; Table 2).

\section{Discussion}

Perioperative BT has been proposed as a possible predictor of poor survival expectance in several malignancies. ${ }^{12-17}$ We tested the hypothesis that intraoperative BT at our center increased a negative effect on survival of patients affected by BCa treated with RC. Our findings confirmed that intraoperative but not postoperative BT is associated with a significant increase of CSM, OM, and disease recurrence. These results were confirmed even after accounting for demographic characteristics and pathological confounders and mainly after accounting for preoperative anemia status and comorbidities, which were advocated as 2 factors that are potentially implicated. Interestingly, no differences were found in terms of survival between postoperative BT and patients who did not receive a BT.

In this context, our data confirm the findings of Abel et $\mathrm{al}^{21}$ who demonstrated in a contemporary population of patients treated with RC between 2002 and 2012, that intraoperative BT has a detrimental effect on survival. Subsequently, the authors confirmed these results with a validation cohort of 1770 patients treated with RC between 1980 and 2005. In this cohort, the 5- and 10-year CSMfree rates for patients who did not receive BT and for patients who underwent postoperative BT were $76 \%$ and $69 \%$ versus $73 \%$ and $67 \%$. However, the 5- and 10-year CSM-free rates for individuals who underwent intraoperative BT and for individuals who underwent intraoperative and postoperative BT were $60 \%$ and $55 \%$ versus $59 \%$ and $51 \%$, respectively. These findings were comparable in our series. Specifically, our 5- and 10-year CSM-free survival rates 


\begin{tabular}{|c|c|c|c|c|c|c|}
\hline \multirow[b]{2}{*}{ Variable } & \multicolumn{2}{|c|}{ Multivariate Recurrence } & \multicolumn{2}{|c|}{ Multivariate CSM } & \multicolumn{2}{|c|}{ Multivariate $\mathbf{O M}$} \\
\hline & HR (95\% Cl) & $\boldsymbol{P}$ & HR (95\% Cl) & $\boldsymbol{P}$ & HR (95\% Cl) & $\boldsymbol{P}$ \\
\hline Age, Years & $1.00(0.98-1.02)$ & .8 & $1.00(0.99-1.02)$ & .5 & $1.01(0.99-1.03)$ & .2 \\
\hline Sex (Ref: Female) & $1.02(0.64-1.62)$ & .9 & $1.23(0.80-.1 .89)$ & .2 & $1.25(0.84-1.86)$ & .2 \\
\hline \multicolumn{7}{|l|}{ CCI } \\
\hline 0 & Ref & Ref & Ref & Ref & Ref & Ref \\
\hline 1 & $1.80(1.10-2.95)$ & .02 & $1.52(0.93-2.48)$ & .1 & $1.58(1.00-2.50)$ & .05 \\
\hline$\geq 2$ & $1.79(1.07-3.01)$ & .03 & $1.60(0.96-2.66)$ & .03 & $2.04(1.28-3.24)$ & .003 \\
\hline PST SM & $1.81(1.02-3.22)$ & .03 & $1.31(0.86-2.45)$ & .1 & $1.25(0.74-2.11)$ & .4 \\
\hline \multicolumn{7}{|l|}{ Pathological Stage } \\
\hline рT0-T2 & Ref & Ref & Ref & Ref & Ref & Ref \\
\hline pT3 & $2.46(1.51-4.01)$ & $<.001$ & $3.36(2.04-5.53)$ & $<.001$ & $2.63(1.75-3.96)$ & $<.001$ \\
\hline pT4 & $2.77(1.48-5.19)$ & $<.001$ & $4.47(2.46-8.11)$ & $<.001$ & $3.73(2.24-6.23)$ & $<.001$ \\
\hline Number of Positive Nodes & $1.06(1.04-1.09)$ & $<.001$ & $1.08(1.05-1.10)$ & $<.001$ & $1.07(1.04-1.10)$ & $<.001$ \\
\hline Number of Nodes Removed & $0.98(0.96-1.94)$ & .03 & $0.97(0.95-0.99)$ & .002 & $0.97(0.96-0.99)$ & .001 \\
\hline Adjuvant Chemotherapy & $1.59(1.08-2.33)$ & .02 & $0.86(0.58-1.29)$ & .5 & $0.76(0.52-1.11)$ & .1 \\
\hline Anemia Status & $0.79(0.55-1.13)$ & .08 & $0.62(0.44-0.88)$ & .01 & $0.67(0.49-0.92)$ & .01 \\
\hline \multicolumn{7}{|l|}{ Receipt of BT } \\
\hline No BT & Ref & Ref & Ref & Ref & Ref & Ref \\
\hline Postoperative BT & $1.50(0.78-2.89)$ & .5 & $1.60(0.81-3.17)$ & .2 & $1.36(0.72-2.60)$ & .4 \\
\hline Intraoperative BT & $1.24(1.03-1.65)$ & .04 & $1.60(1.20-2.26)$ & .02 & $1.45(1.02-2.08)$ & .03 \\
\hline
\end{tabular}

Abbreviations: $\mathrm{BT}=$ blood transfusion; $\mathrm{CCI}=$ Charlson comorbidity Index; CSM = cancer-specific mortality; HR = hazard ratio; OM = overall mortality; PSTSM = positive soft tissue surgical margin; Ref $=$ reference.

were $64 \%$ and $52 \%$ versus $60 \%$ and $60 \%$ for patients without BT and for patients who underwent postoperative BT. Although 5- and 10-year CSM-free survival rates for men who received intraoperative BT and who underwent intraoperative and postoperative BT, were $49 \%$ and $42 \%$ versus $50 \%$ and $40 \%$, respectively. Others previous investigators assessed the association between BT and oncologic outcomes in the RC population. In this context, Morgan et $\mathrm{al}^{9}$ in their study assessed the effect of BT and survival in a population with a limited follow-up (median, 25 months) and did not account for preoperative $\mathrm{Hb}$ levels. Kluth et $\mathrm{al}^{11}$ failed to report an association between BT and survival in a numerous multicentric cohort. Gierth et $\mathrm{al}^{10}$ described in a monocentric series of 350 patients with RC a detrimental effect of BT on survival after accounting for anemia status as a confounder in a multivariable model. Finally, Linder et $\mathrm{al}^{8}$ presented a report on the largest cohort available in the literature on 2060 RC patients, with a median follow-up of 10.9 years. They demonstrated that perioperative BT is associated with increased risk of postoperative tumor recurrence, CSM, and OM after RC even after accounting for all available preoperative confounders. Of note, these studies were limited by the absence of stratification between intraoperative and postoperative $\mathrm{BT},{ }^{8-11}$ and by short follow-up. 9,10

From a biological standpoint, several theories could explain the association between BT and the detrimental effect on survival after surgery. First, the immunosuppressive effect of BT was previously described in the literature as related to transfusion-related immune modulation. ${ }^{18}$ Immune modulation is mediated by suppression of cytotoxic cell and monocyte activity, release of immunosuppressive prostaglandins, inhibition of interleukin-2 production, and increase in suppressor of T-cell activity. ${ }^{24}$ Second, transfusion-induced anergy due to the presentations of large amounts of antigens during BT could also explain these results; this type of effect was used therapeutically to reduce renal allograft rejection before immunosuppressant drugs become available. It was also described as a transfusion-induced anergy due to the presentations of large amounts of antigen during PBT. ${ }^{25}$ This type of effect is used therapeutically to reduce renal allograft rejection when immunosuppressant drugs become available. ${ }^{26}$ However, at the moment no consensus has been reached about the real effect of BT on survival and further studies are needed to better understand the mechanisms of this effect. More specifically, considering intraoperative BT, further theories advocated consist of an increased spread of tumor cells caused by surgical manipulation ${ }^{20}$ or an association between a decreased host immunity caused by anesthetics and opioids that can have a synergic effect with cancer manipulation. ${ }^{19}$

The importance of our study in relation to previous reports consists of several aspects. First, our investigation represents the first confirmation of the findings of Abel et $\mathrm{al}^{21}$ using a large European single-institution cohort. This is fundamental considering the clinical implications that can be applied to these patients. Second, our report confirms in a monocentric setting that physicians should consider timing as a crucial parameter when BT is indicated.

Despite several strengths, our study is not devoid of limitations. First and foremost we recognize that our study is limited by its observational nature, and thus our results should be interpreted within the limits of its retrospective design. For example, the 
administration of BT was not standardized overall and was left to the clinical judgement of each treating physician. However, we partially addressed this limitation considering the monocentric status of our report, and all the parameters considered by the physicians in the decision for administration of BT were taken into account in multivariable analyses. Moreover, no comparisons were made considering autologous compared with allogeneic transfusion. However, previous retrospective and prospective series in prostate ${ }^{16}$ or rectal $^{27}$ cancers did not show any benefit from autologous transfusion compared with standard allogeneic transfusion.

\section{Conclusion}

We use a large single-institution series with long-term follow-up to confirm for the first time that intraoperative, but not postoperative BT, are related to a detrimental effect on survival in patients affected by BCa who undergo RC. These results should be taking into account by physicians to administer PBT according to the correct timing.

\section{Clinical Practice Points}

- Transfusion has been related to poor survival after major surgeries in several cancers. Recently, timing of transfusion has been advocated as crucial in determining this connection as a consequence of their immunosuppressive effect and/or the related increased intraoperative cancer manipulation.

- Our investigation demonstrated that intraoperative but not postoperative transfusion are related to poor survival after RC in BCa patients.

- These observations highlight the importance of timing in the administration of transfusion in BCa patients. Physicians should avoid transfusion intraoperatively.

\section{Disclosure}

The authors have stated that they have no conflicts of interest.

\section{References}

1. Witjes JA, Compérat E, Cowan NC, et al. EAU guidelines on muscle-invasive and metastatic bladder cancer: summary of the 2013 guidelines. Eur Urol 2014; 65: 778-92.

2. Shariat SF, Karakiewicz PI, Palapattu GS, et al. Outcomes of radical cystectomy for transitional cell carcinoma of the bladder: a contemporary series from the Bladder Cancer Research Consortium. I Urol 2006; 176:2414-22, discussion 2422.

3. Todenhöfer T, Renninger M, Schwentner C, Stenzl A, Gakis G. A new prognostic model for cancer-specific survival after radical cystectomy including pretreatment thrombocytosis and standard pathological risk factors. BJU Int 2012; 110:E533-40.
4. Hermanns T, Bhindi B, Wei Y, et al. Pre-treatment neutrophil-to-lymphocyte ratio as predictor of adverse outcomes in patients undergoing radical cystectomy for urothelial carcinoma of the bladder. Br I Cancer 2014; 111:444-51.

5. Gakis G, Todenhöfer T, Stenzl A. The prognostic value of hematological and systemic inflammatory disorders in invasive bladder cancer. Curr Opin Urol 2011; 21:428-33.

6. Moschini M, Suardi N, Pellucchi F, et al. Impact of preoperative thrombocytosis on pathological outcomes and survival in patients treated with radical cystectomy for bladder carcinoma. Anticancer Res 2014; 34:3225-30.

7. Viers BR, Boorjian SA, Frank I, et al. Pretreatment neutrophil-to-lymphocyte ratio is associated with advanced pathologic tumor stage and increased cancer-specific mortality among patients with urothelial carcinoma of the bladder undergoing radical cystectomy. Eur Urol 2014; 66:1157-64.

8. Linder BJ, Frank I, Cheville JC, et al. The impact of perioperative blood transfusion on cancer recurrence and survival following radical cystectomy. Eur Urol 2013; 63:839-45.

9. Morgan TM, Barocas DA, Chang SS, et al. The relationship between perioperative blood transfusion and overall mortality in patients undergoing radical cystectomy for bladder cancer. Urol Oncol 2013; 31:871-7.

10. Gierth M, Aziz A, Fritsche HM, et al. The effect of intra- and postoperative allogeneic blood transfusion on patients' survival undergoing radical cystectomy for urothelial carcinoma of the bladder. World J Urol 2014; 32:1447-53.

11. Kluth LA, Xylinas E, Rieken M, et al. Impact of peri-operative blood transfusion on the outcomes of patients undergoing radical cystectomy for urothelial carcinoma of the bladder. BJU Int 2014; 113:393-8.

12. Fein DA, Lee WR, Hanlon AL, et al. Pretreatment hemoglobin level influences local control and survival of T1-T2 squamous cell carcinomas of the glottic larynx. I Clin Oncol 1995; 13:2077-83.

13. Beresford MJ, Burcombe R, Ah-See ML, Stott D, Makris A. Pre-treatment haemoglobin levels and the prediction of response to neoadjuvant chemotherapy in breast cancer. Clin Oncol (R Coll Radiol) 2006; 18:453-8.

14. Qiu M, Yuan Z, Luo H, et al. Impact of pretreatment hematologic profile on survival of colorectal cancer patients. Tumour Biol 2010; 31:255-60.

15. Yeoh TY, Scavonetto F, Weingarten TN, et al. Perioperative allogeneic nonleukoreduced blood transfusion and prostate cancer outcomes after radical prostatectomy. Transfusion 2014; 54:2175-81.

16. Chalfin HJ, Frank SM, Feng Z, et al. Allogeneic versus autologous blood transfusion and survival after radical prostatectomy. Transfusion 2014; 54:2168-74.

17. Vamvakas EC. Allogeneic blood transfusion and cancer recurrence: 20 years later. Transfusion 2014; 54:2149-53.

18. Vamvakas EC, Blajchman MA. Transfusion-related immunomodulation (TRIM): an update. Blood Rev 2007; 21:327-48.

19. Colvin LA, Fallon MT, Buggy DJ. Cancer biology, analgesics, and anaesthetics: is there a link? Br J Anaesth 2012; 109:140-3.

20. Pesta M, Fichtl J, Kulda V, Topolcan O, Treska V. Monitoring of circulating tumor cells in patients undergoing surgery for hepatic metastases from colorectal cancer. Anticancer Res 2013; 33:2239-43.

21. Abel EJ, Linder BJ, Bauman TM, et al. Perioperative blood transfusion and radical cystectomy: does timing of transfusion affect bladder cancer mortality? Eur Urol 2014; 66:1139-47.

22. Edge SB, Compton CC. The American Joint Committee on Cancer: the 7 th edition of the AJCC cancer staging manual and the future of TNM. Ann Surg Oncol 2010; 17:1471-4.

23. World Health Organization. Haemoglobin concentrations for the diagnosis of anaemia and assessment of severity. VMNIS | Vitamin and Mineral Nutrition Information System. Available at: http://www.who.int/vmnis/indicators/ haemoglobin.pdf. Accessed May 20, 2015.

24. Vamvakas EC. Possible mechanisms of allogeneic blood transfusion-associated postoperative infection. Transfus Med Rev 2002; 16:144-60.

25. Blumberg N, Heal JM. Effects of transfusion on immune function. Cancer recurrence and infection. Arch Pathol Lab Med 1994; 118:371-9.

26. Opelz G, Terasaki PI. Improvement of kidney-graft survival with increased numbers of blood transfusions. N Engl J Med 1978; 299:799-803.

27. Harlaar JJ, Gosselink MP, Hop WC, Lange JF, Busch OR, Jeekel H. Blood transfusions and prognosis in colorectal cancer: long-term results of a randomized controlled trial. Ann Surg 2012; 256:681-6, discussion 686-7. 